\title{
Causes of perinatal hypoxia during resuscitation in premature newborns with low and extremely-low body weight
}

Immanuel Kant Baltic Federal

University

\section{Svetlana Perepelitsa ${ }^{1,2}$, Arkady Goloubev ${ }^{1}$, Artem Kuzovlev ${ }^{1}$}

1 V.A. Negovsky Research Institute of General Reanimatology,

Federal Research and Clinical Center of Intensive Care Medicine and Rehabilitology, Moscow, Russian Federation

2 Imannuel Kant Baltic Federal University, Kaliningrad, Russian Federation

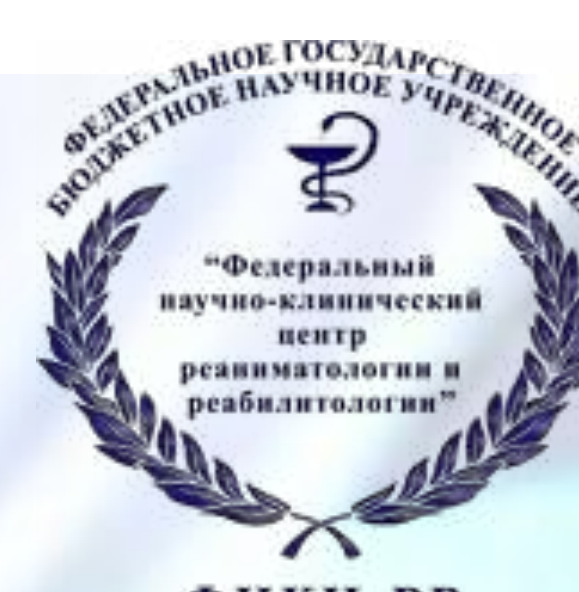

ФНКЦ РР
The aim of the study was to identify the causes of perinatal hypoxia in the deep-preterm neonates.

Materials and methods. The analysis of causes of deaths of 23 premature newborns was done. Average gestational age of newborns was $26.4 \pm 2.7$ weeks, birth weight was $972.4 \pm 355.8$ grams. $78.3 \%$ of the newborns had an extremely low body weight. Since birth newborns has undergone a comprehensive treatment and several modes of mechanical ventilation. Treatment did not contribute to the normalization of metabolic disorders (acidosis, hyperlactatemia, hypoxemia) which led to the development of multi-organ failure and death.

Results. At birth neonates revealed signs of hypoxia: low Apgar score and decompensated mixed metabolic acidosis, hypoxemia. Resuscitation failed, the second Apgar score remained low, which confirmed the severity of perinatal hypoxia and the weak response of the newborn to ongoing primary resuscitation. Analysis of the main causes of deaths in premature neonates showed that the main cause of death were congenital infections in $52 \%$. The more massive was the inflammation, the heavier was the hypoxia at birth and the weaker was the response of the newborn to ongoing treatment.

Conclusions. Severe perinatal hypoxia, manifested by a very low Apgar score and laboratory criteria (hypoxemia and decompensated lactate acidosis), resistant to standard resuscitation is associated with the development of intrauterine infection in premature neonates. Hypoxemia and decompensated metabolic acidosis, which persist in the first hours of postnatal life, indicate the severity of intrauterine lung injury of an infectious nature.

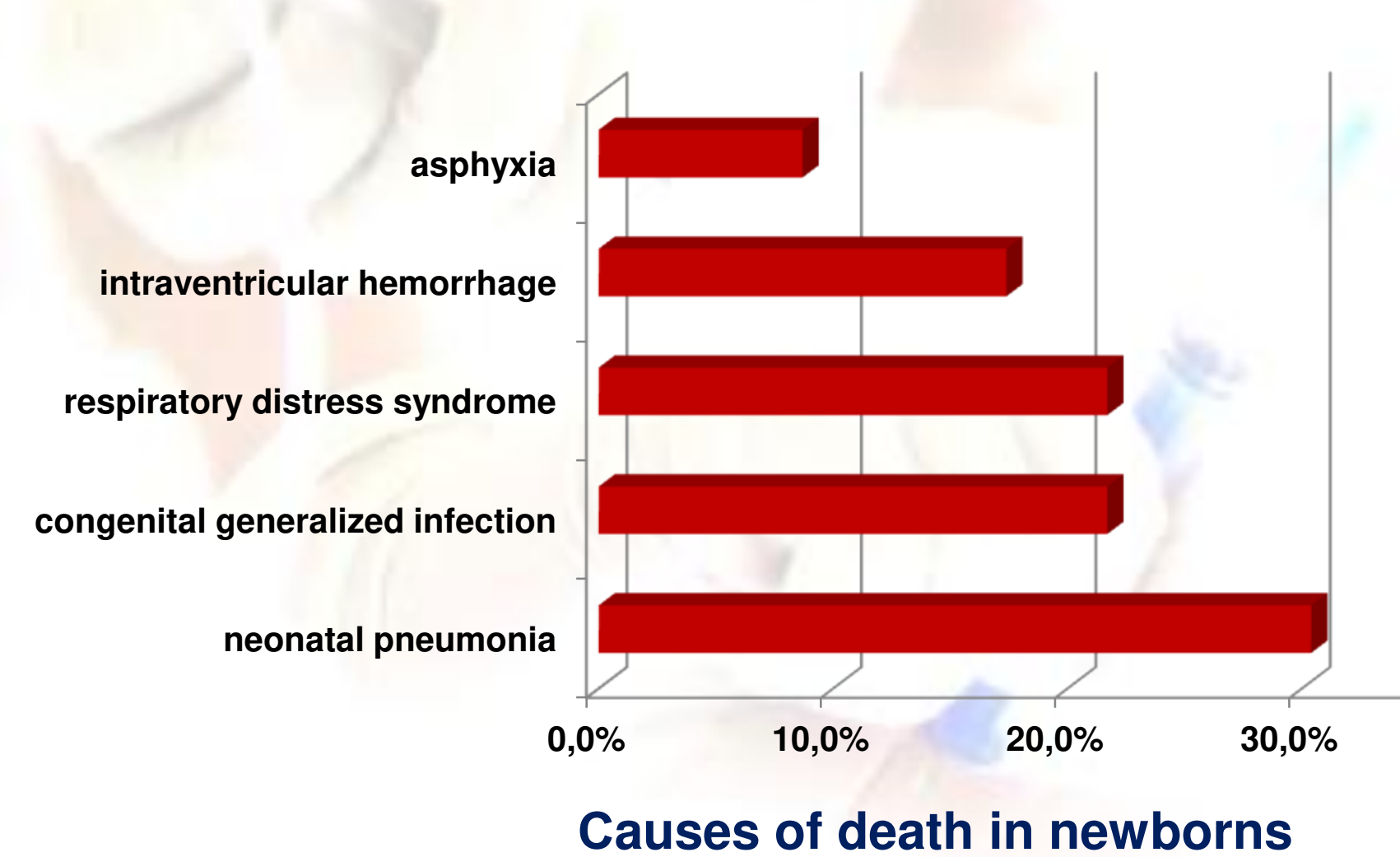

Causes of death in newborns

$$
\mathrm{pH}=6,9-7,14
$$

$B E=-15,6-16,7 \mathrm{mmol} / \mathrm{l}$

\section{Lactate $=7,5-10 \mathrm{mmol} / \mathrm{l}$}

At birth, decompensated metabolic acidosis was detected

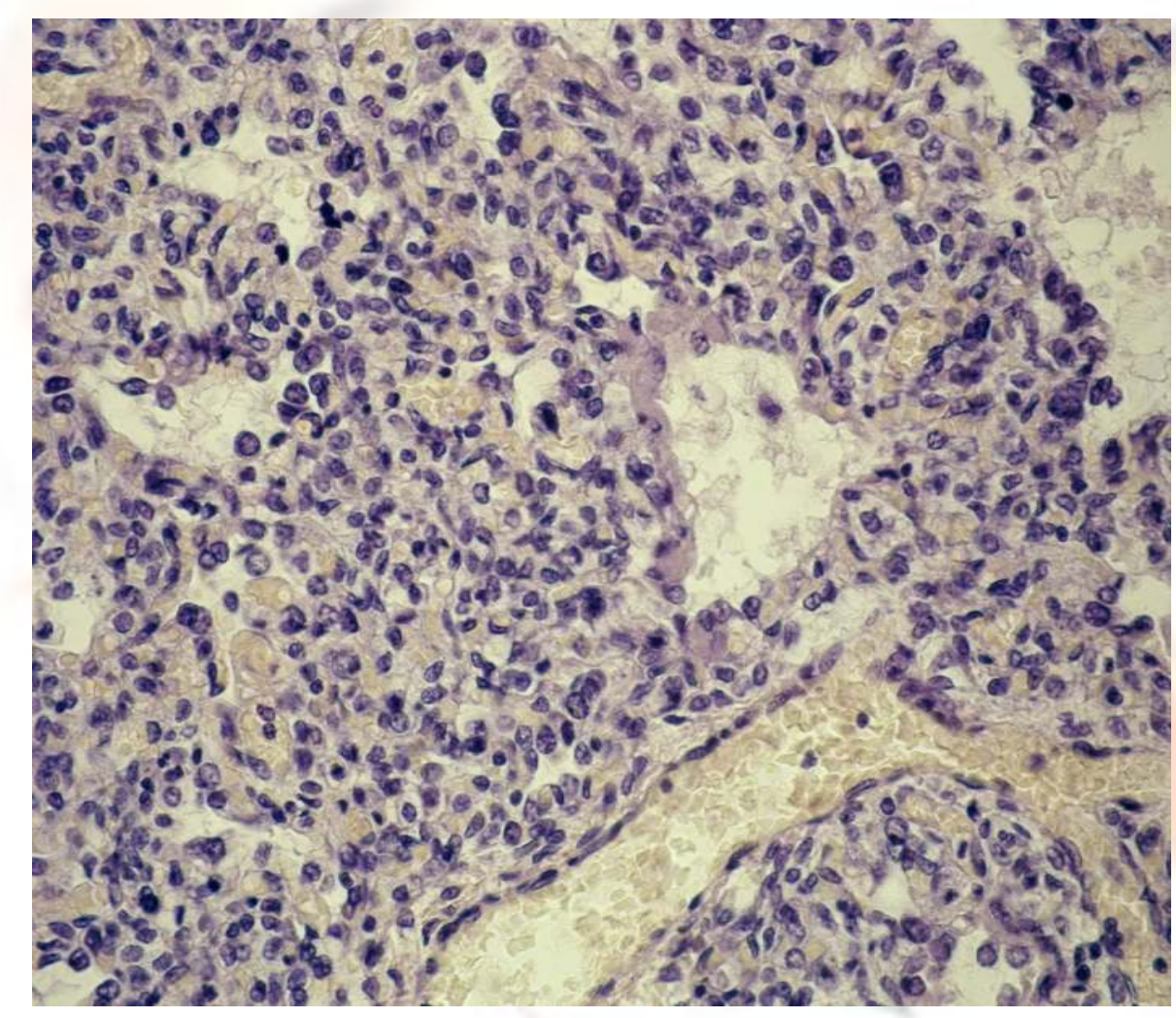

Fig. 2. Congenital pneumonia and hyaline membranes in a preterm neonate. Hematoxylin-eosin. X1000

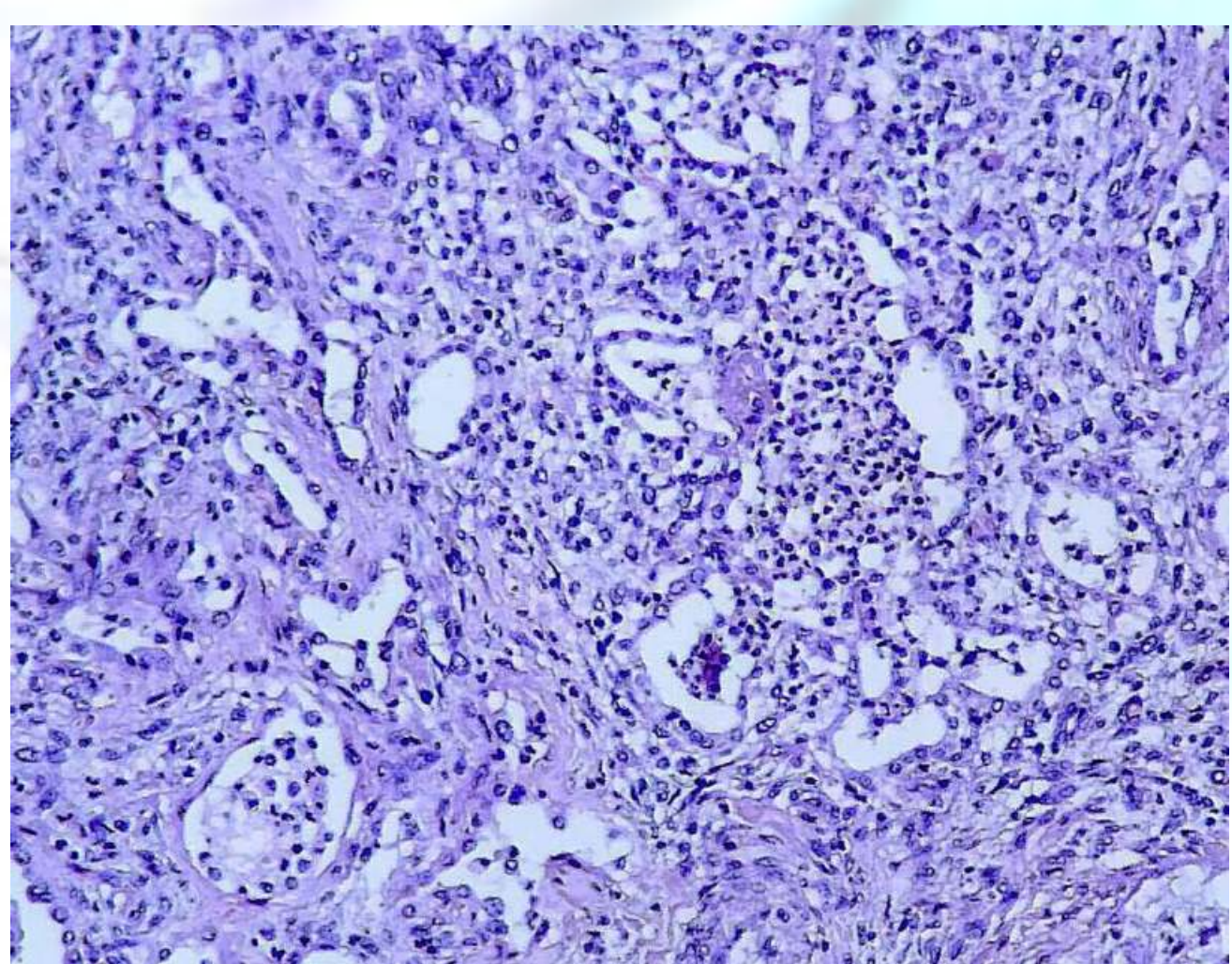

Fig. 1. Congenital pneumonia in a premature newborn: diffuse-focal infiltration of interalveolar septa, alveoli are filled with segmented leukocytes, alveolar macrophages. Hematoxylin-eosin. X1000.

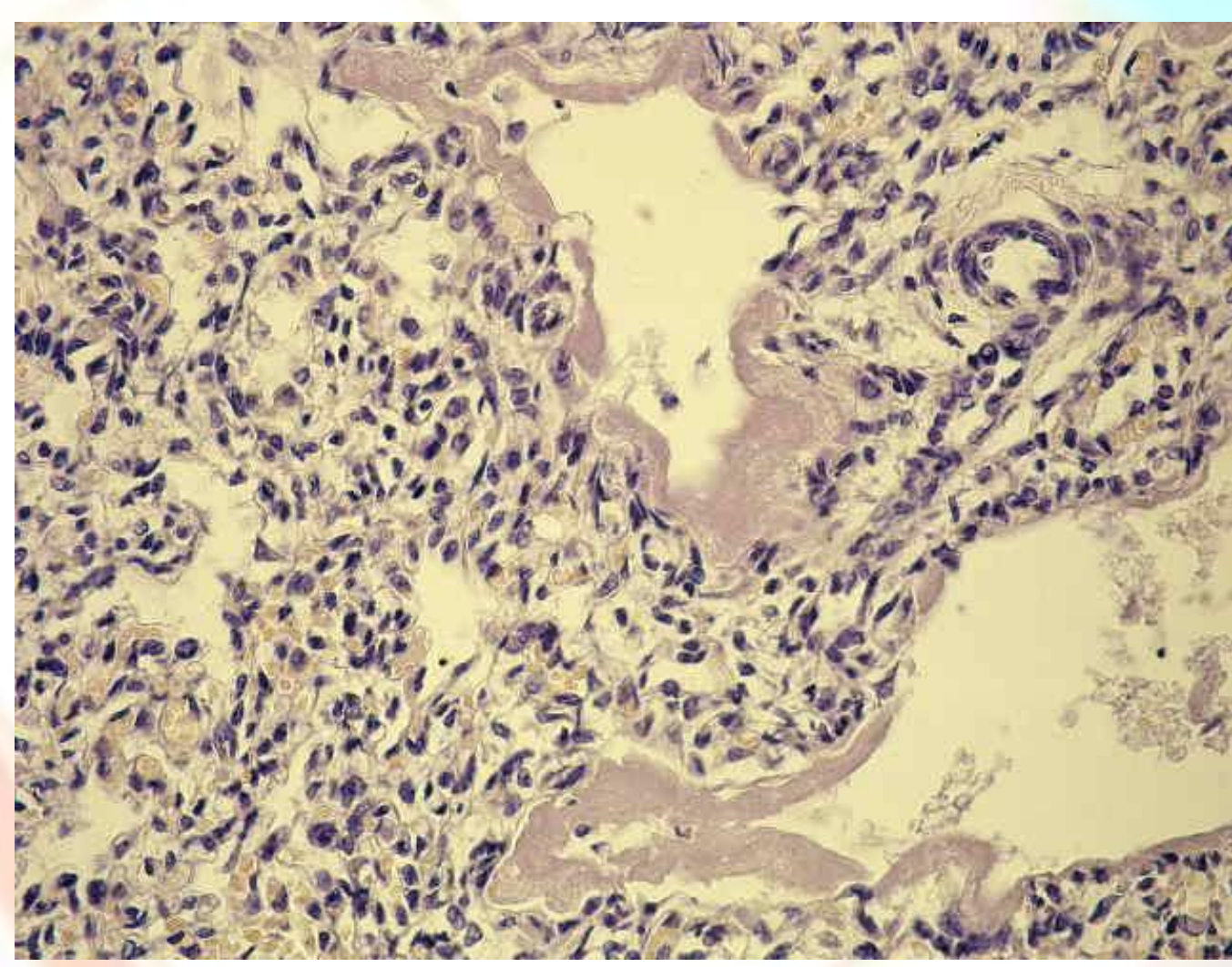

Fig. 3. Hyaline membranes of various forms and localizations. Hematoxylin-eosin. X1000 